\title{
Morfologia de ovos de Triatoma circummaculata e Triatoma rubrovaria (Hemiptera, Reduviidae)
}

Egg morphology of Triatoma circummaculata and Triatoma rubrovaria (H emiptera, Reduviidae)

\section{João A da Rosaa, José Maria S Baratab, Jair Lício F Santos ${ }^{b}$ e Mario Cilensec}

aD epartamento de Ciências Biológicas da Faculdade de Ciências Farmacêuticas da U niversidade Estadual Paulista (U nesp). A raraquara, SP, Brasil. 'b epartamento de Epidemiologia da Faculdade de Saúde Pública da Universidade de São Paulo. São Paulo, SP, Brasil. 'D epartamento de Físico-Q uímica do Instituto de Q uímica da U nesp. Araraquara, SP, Brasil 


\title{
Morfologia de ovos de Triatoma circummaculata e Triatoma rubrovaria (Hemiptera, Reduviidae)* Egg morphology of Triatoma circummaculata and Triatoma rubrovaria (Hemiptera, Reduviidae)
}

\author{
João A da Rosaa, José Maria S Baratab, Jair Lício F Santos ${ }^{\mathrm{b}}$ Mario Cilense \\ aD epartamento de Ciências Biológicas da Faculdade de Ciências Farmacêuticas da Universidade

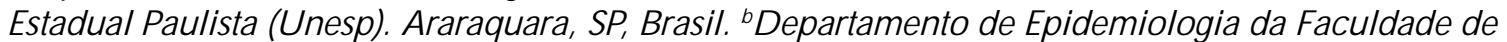 \\ Saúde Pública da Universidade de São Paulo. São Paulo, SP, Brasil. 'D epartamento de Físico-Q uímica \\ do Instituto de Q uímica da U nesp. Araraquara, SP, Brasil
}

\section{Descritores}

Triatoma, ultraestrutura\# . Ovos, análise ${ }^{\#}$. Morfologia ${ }^{\#}$. - Embrião.

\section{Keywords}

Triatoma, ultrastructure ${ }^{\#}$. Eggs, analysis ${ }^{\#}$. Morphology $y^{\#}$ - Embryo.

\section{Resumo}

Objetivo

Estudar morfologicamente ovos de T. circummaculata e T. rubrovaria.

Métodos

Quarenta ovos das duas espécies foram mensurados por meio de projetor de perfil Nikon modelo 6C, e utilizado o teste Student, para execução da análise estatística. As observações morfológicas foram feitas em microscópio eletrônico de varredura JEOL, modelo JSM-T330A.

Resultados/Conclusões

A análise estatística revelou que os ovos e as cascas de ovos de T. rubrovaria são significativamente maiores que os de T. circummaculata. Observações microscópicas mostraram que a borda corial, assim como a goteira espermática, são bem mais evidentes nos ovos de T. rubrovaria que nos de T. circummaculata. $\mathrm{O}$ exocório das duas espécies é formado por áreas poligonais, que são hexagonais em sua maioria. Foram observados também o momento da eclosão do embrião e os detalhes das exúvias embrionárias.

\footnotetext{
Abstract

Objective

To study morphologically the eggs of T. circummaculata and T. rubrovaria. Methods

Forty eggs of the two species were measured through Nikon model 6C profile projector. Student test was utilized for the statistical analysis and scanning electron microscopy for the morphological study of the eggs.

\section{Results/Conclusions}

The statistical analysis shows that the T. rubrovaria eggs are larger that those of $\mathrm{T}$. circummaculata. Microscopical observations revealed that the chorial rim and the spermatic groove are less evident in T. circummaculata than in T. rubrovaria. Both species, the majority of exochorion cells are hexagonal. It was also observed the embryo eclosion and details of the embryonic molt.
}

Correspondência para/Correspondence to: João Aristeu da Rosa

Rodovia Araraquara-Jaú Km1

14801-902 Araraquara, SP, Brasil

E-mail: rosaja@boldo.fcfar.unesp.br
*Financiado pela Fundaça para o Desenvolvimento, Universidade Estadual Paulista (Processo n. 530/90-DFP) Parte da tese de doutorado apresentada ao Instituto de Ciências Biomédicas da Universidade de São Paulo, 1995 Edição subvencionada pela Fapesp (Processo no 00/01601-8).

Recebido em 22/2/1999. Reapresentado em 6/6/2000. Aprovado em 27/6/2000. 


\section{IN TRO DU ÇÃO}

Triatoma circummaculata e T. rubrovaria são espécies de Triatominae que ocupam ecótopos semelhantes e por vezes podem ser encontradas juntas (Abalos \& Wygodzinsky, ${ }^{1}$ 1951; Lent \& Wygodzinsky, ${ }^{11}$ 1979). Segundo Abalos \& Wygodzinsky, ${ }^{1}$ Carcavallo \& Martinez $^{4}$ (1985) e Carcavallo et $\mathrm{al}^{5}$ (1988), $T$. rubrovaria pode ser encontrada nas províncias de Corrientes, Entre Rios e Missiones, na Argentina. Está distribuído em todo Uruguai, de acordo com Franca Rodrigues et $\mathrm{al}^{6}$ (1980). As áreas de ocorrência de $T$. rubrovaria no Brasil localizavam-se nos Estados do Paraná e Rio Grande do Sul, segundo Lent \& Wygodzinsky ${ }^{11}$ (1979), mas atualmente restringem-se à área endêmica de doença de Chagas no Rio Grande do Sul, de acordo com Rosa ${ }^{15}$ (1995) (Figura 1).

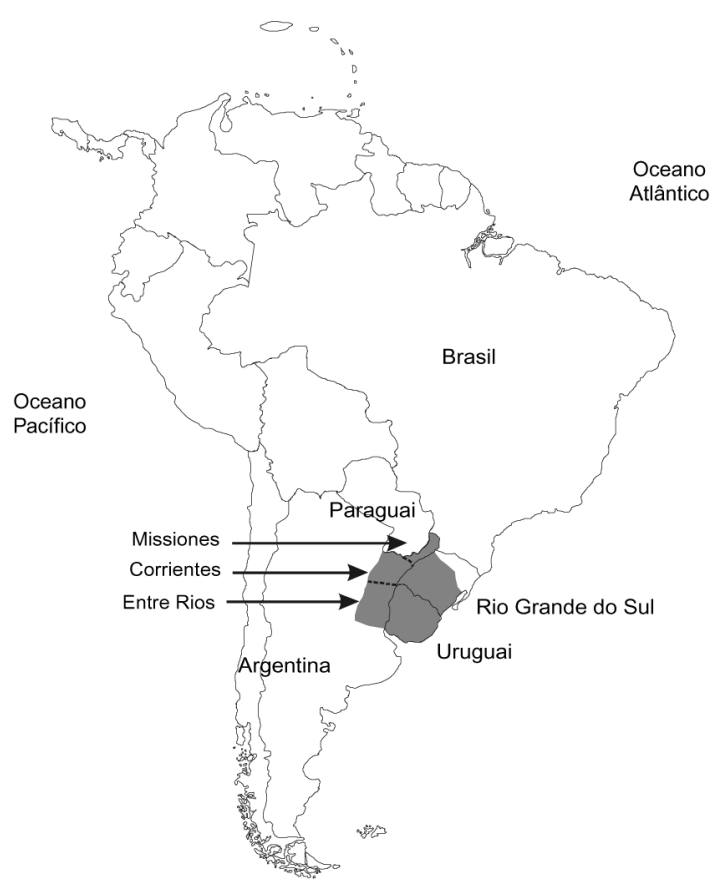

Figura 1 - Área de ocorrência de Triatoma circummaculata e Triatoma rubrovaria na América do Sul.

A distribuição de $T$. circummaculata está restrita ao Rio Grande do Sul no Brasil e ao Uruguai (Figura 1), segundo Abalos \& Wygodzinsky ${ }^{1}$ (1951), Lent \& Wygodzinsky $^{11}$ (1979) e Carcavallo \& Martinez $^{4}$ (1985).

A importância de T. rubrovaria quanto à veiculação de T. cruzi ao homem é um fato comprovado na literatura (Silva, ${ }^{16}$ 1985; Perlowagora-Szumlewicz, ${ }^{13}$ 1988). Segundo Franca Rodriguez et $\mathrm{al}^{6}$ (1980), T. circummaculata é a segunda espécie em importância no Uruguai. Por outro lado, o número de exemplares coletados de $T$. rubrovaria (568) e de T. circummaculata (258) no Rio Grande do Sul, assim como a existência de residências da área rural próximas ao ecótopo silvestre, corroboram a importância epidemiológica das duas espécies (Rosa, ${ }^{15}$ 1995).

Os trabalhos sobre ovos de Triatominae têm colaborado principalmente para a distinção genérica ou específica dessa subfamília (Galliard, ${ }^{7}$ 1936; Lent \& Wygodzinsky, ${ }^{11}$ 1979; Barata, ${ }^{3}$ 1981; Jurberg et al, ${ }^{10}$ 1986).

Visando a ampliar o conhecimento sobre os Triatominae e contribuir para a caracterização de $T$. circummaculata e $T$. rubrovaria, foram efetuadas mensurações de ovos e cascas de ovos e estudo morfológico por microscopia eletrônica de varredura das exúvias embrionárias e dos ovos, provenientes de exemplares coletados no ambiente silvestre (Rosa, ${ }^{15}$ 1995). Foi também elaborada figura para mostrar a distribuição das duas espécies.

\section{MÉTODOS}

Os ovos foram obtidos de exemplares capturados em cinco coletas realizadas nos municípios de Caçapava do Sul, São Francisco de Assis e São Sepé, que fazem parte da área endêmica de doença de Chagas no Rio Grande do Sul, no período de dezembro de 1990 a abril de 1992.

Os ovos e as cascas foram lavados em água destilada, com auxílio de aparelho ultra-som, desidratados em série alcoólica e secos em estufa a $40-50^{\circ}$ por 20 min. Os demais procedimentos estão descritos em Rosa et $\mathrm{al}^{14}$ (1992). O estudo morfológico foi conduzido em microscópio eletrônico de varredura $\mathrm{Jeol}$, modelo JSM-T330 A.

Foram mensurados 40 ovos e 40 cascas de ovos de cada uma das espécies: T. circummaculata e $T$. rubrovaria. Os ovos das duas espécies foram mensurados embrionados, mas em diferentes estádios de evolução. O aparelho utilizado para essas mensurações foi o Projetor de Perfil Nikon, modelo 6C.

Foram mensurados, por uma única pessoa, o comprimento e a largura dos ovos e das cascas de ovos. Foi executada somente uma medida de comprimento e de largura de cada amostra.

Foi utilizado o teste Student para analisar a diferença estatística entre duas amostras não pareadas ou independentes, que poderiam ser ou não do mesmo tamanho. Para tanto, seguiram-se as instruções de

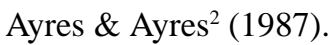




\section{RESULTADOS}

Os ovos de $T$. circummaculata apresentam 1,55 mm em média de comprimento e $0,90 \mathrm{~mm}$ em média de largura. As cascas de ovos medem 1,46 mm em média de comprimento e $0,87 \mathrm{~mm}$ de largura. Os ovos de $T$. rubrovaria possuem $1,85 \mathrm{~mm}$ em média de comprimento e 1,14 mm de largura. As cascas de ovos medem $1,77 \mathrm{~mm}$ em média de comprimento e 1,16 mm de largura (Tabela1).

Tanto o opérculo do ovo de T. circummaculata como o de $T$. rubrovaria apresentam-se externamente sob a forma circular convexa, sendo constituídos por numerosas áreas poligonais irregulares de superfície estofada, com perfurações distribuídas aleatoriamente. As linhas que dividem as áreas poligonais da superfície opercular também apresentam perfurações e/ ou ranhuras (Figuras 2a e b, 3b).

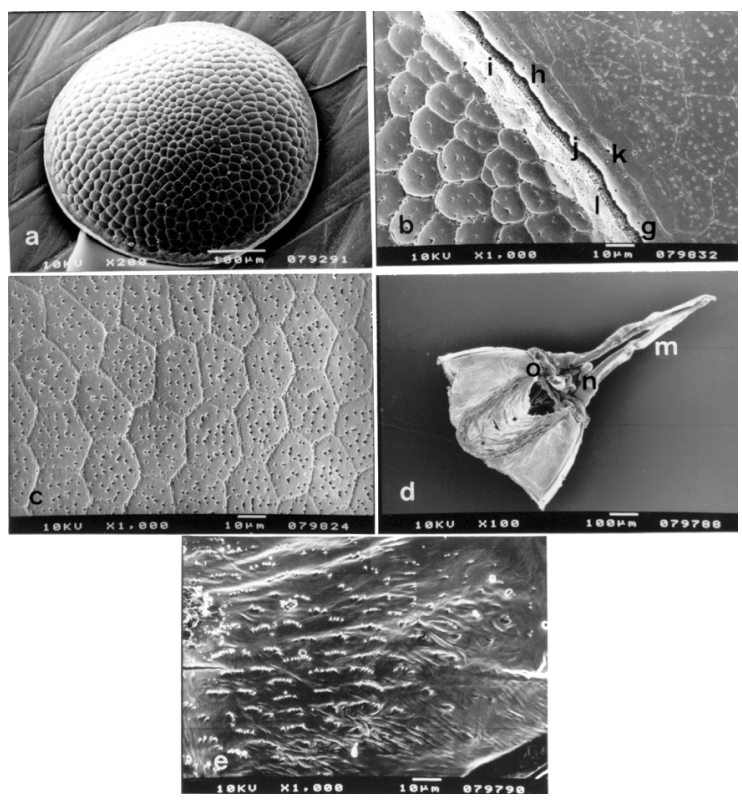

$a=$ opérculo; $b=$ opérculo e exocório; $c=$ exocório; $d=$ exúvia de embrião; e= detalhe de exúvia de embrião; $g=$ goteira espermática; $\mathrm{h}=$ aerópilas; $\mathrm{i}=$ borda opercular; $\mathrm{j}=$ borda corial $\mathrm{k}=$ micrópila; $\mathrm{I}=$ faixa de vedação; $\mathrm{m}=$ antena; $\mathrm{n}=$ labro; $\mathrm{o=}$ pernas em formação.

Figura 2 - O vo de T. circummaculata, por meio de MEV.

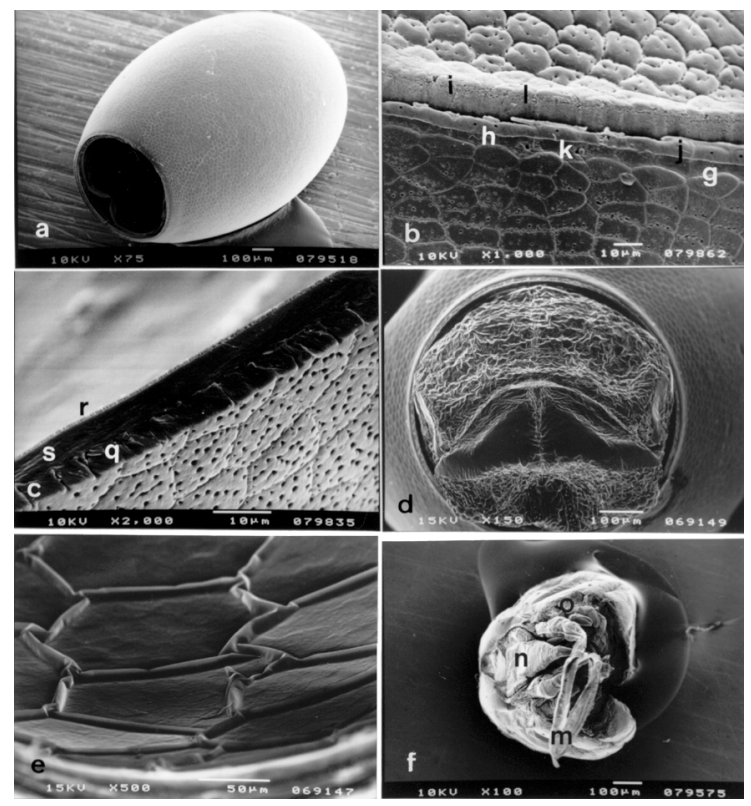

$a=$ casca de ovo; $b=$ opérculo e exocório; $c=$ corte transversal da casca; $d=$ eclosão de ninfa; e= película interna de casca de ovo; $f=$ exúvia de embrião; $g$ = goteira espermática; $h=$ aerópilas; $\mathrm{i}=$ borda opercular; $\mathrm{j}=$ borda corial; $\mathrm{k}=$ micrópila; $\mathrm{I}=$ faixa de vedação; $m=$ antena; $n=$ labro; $0=$ pernas em formação; $q=$ camada externa; $r=$ camada interna; $s=$ camada média.

Figura 3 - O vo de T. rubrovaria, por meio de MEV.

A linha opercular limitante é constituída por massa cementante, denominada faixa de vedação, responsável pela fixação do opérculo à borda corial do ovo (Figuras 2b, 3b).

A superfície externa do exocório do ovo das duas espécies apresenta-se dividida em áreas poligonais, a maioria delas hexagonais, com numerosas perfurações (Figuras 2c, 3a, b e c). A superfície interna da casca do ovo mostra áreas poligonais lisas, delimitadas por pregas e sensivelmente maiores que as externas (Figura 3e).

No limite superior do ovo percebe-se a borda corial, que apresenta no plano anterior a goteira espermática, onde se situam as micrópilas; no plano posterior encontram-se as aerópilas (Figuras 2b, 3b).

Tabela 1 - Resultados estatísticos das mensurações (em mm) de 40 ovos e 40 cascas de ovos de T. circummaculata e T. rubrovaria.

\begin{tabular}{|c|c|c|c|c|c|c|c|c|}
\hline & \multicolumn{4}{|c|}{ T. circummaculata } & \multicolumn{4}{|c|}{ T. rubrovaria } \\
\hline & \multicolumn{2}{|c|}{ O vo } & \multicolumn{2}{|c|}{ Casca } & \multicolumn{2}{|c|}{ O vo } & \multicolumn{2}{|c|}{ Casca } \\
\hline & $\mathrm{C}$ & L & $\mathrm{C}$ & $L$ & C & $L$ & C & L \\
\hline $\begin{array}{l}\text { Média } \\
\text { Desvio-padrão }\end{array}$ & $\begin{array}{r}1,55 \\
0,076\end{array}$ & $\begin{array}{l}0,905 \\
0,029\end{array}$ & $\begin{array}{l}1,467 \\
0,067\end{array}$ & $\begin{array}{l}0,872 \\
0,031\end{array}$ & $\begin{array}{l}1,856 \\
0,083\end{array}$ & $\begin{array}{l}1,143 \\
0,045\end{array}$ & $\begin{array}{l}1,777 \\
0,080\end{array}$ & $\begin{array}{l}1,169 \\
0,010\end{array}$ \\
\hline
\end{tabular}

$\mathrm{C}=$ comprimento

$\mathrm{L}=$ largura 
O corte transversal da casca permitiu observar que o cório é constituído por três camadas: uma externa, em que se situam canais que darão ao meio externo através de perfurações, uma camada média compacta e uma camada interna porosa estreita (Figura 3c).

A primeira porção do embrião que sai do ovo é a região compreendida entre o tórax e a cabeça na altura dos olhos (Figura 3d). No momento da eclosão o embrião libera uma exúvia hemisférica. Nessa exúvia percebemse as antenas, o labro e os três pares de pernas em formação (Figuras 2d, 2e, 3f).

\section{DISCUSSÃO}

Embora os ovos de Triatominae tenham sido estudados por diversos autores, entre os quais Galliard ${ }^{7}$ (1936) e Lent \& Wygodzinsky ${ }^{11}$ (1979), poucos utilizaram as estruturas morfológicas dos ovos para a distinção específica. Entre os que as utilizaram podem ser citados:

- Barata $^{3}$ (1981), estudando características de ovos de dez espécies de Rhodnius, elaborou chaves dicotômicas que permitem a distinção específica do citado gênero;

- Gonçalves et $\mathrm{al}^{8}$ (1985) descreveram diferenças morfológicas entre ovos de $T$. maculata e $T$. pseudomaculata;

- Haridass $^{9}$ (1986), trabalhando com Triatominae e Echtrichodiinae, observou diferenças entre os ovos dessas subfamílias de Ruduviidae.

No presente trabalho, a mensuração de amostras de ovos e de cascas de ovos (Figura 3a) e a posterior análise estatística mostraram que os ovos e as cascas de ovos de $T$. rubrovaria são significativamente maiores que os de $T$. circummaculata (Tabelas 1,2).

A análise estatística também revelou que os ovos de T. circummaculata são significativamente maiores que as respectivas cascas, enquanto os ovos de T. rubrovaria o são apenas quanto ao comprimento (Tabela 2).

A textura do exocório do opérculo do ovo de T. circummaculata e T. rubrovaria são semelhantes à de T. maculata e de $T$. brasiliensis, conforme pode ser verificado nas publicações de Gonçalves et $\mathrm{al}^{8}$ (1985) e de Jurberg et $\mathrm{al}^{10}$ (1986), respectivamente.
A borda corial do ovo de $T$. circummaculata e de $T$. rubrovaria apresenta semelhança com $T$. maculata, descrita por Gonçalves et $\mathrm{al}^{8}$ (1985), mas não possui colo e colarinho, que são estruturas situadas na extremidade opercular dos ovos de Rhodnius, conforme descrito por $\operatorname{Barata}^{3}$ (1981).

A presença de três camadas no cório do corpo do ovo de T. rubrovaria observada no presente estudo, já havia sido observada anteriormente em ovos de Rhodnius, por $\operatorname{Barata}^{3}$ (1981).

Além da diferença significativa quanto ao tamanho dos ovos e das cascas de ovos de T. circummaculata e $T$. rubrovaria, foram percebidas duas outras diferenças morfológicas entre essas duas espécies. A borda corial, assim como a goteira espermática, são bem mais evidentes nos ovos de $T$. rubrovaria que nos de $T$. circummaculata (Figuras 2b, 3b).

Quanto às exúvias embrionárias, mostradas no presente estudo (Figuras 2d, 2e, 3f), já foram referidas anteriormente por Williams \& Buxton ${ }^{17}$ (1916), que observaram mudas do embrião dos Orthoptera e de outras ordens de inseto, e por Galliard ${ }^{7}$ (1936), que descreveu a exúvia do embrião de $T$. vitticeps. César Pinto foi quem observou a liberação da exúvia antes da eclosão do embrião, conforme citado por Galliard. $^{7}$

Mellanby ${ }^{12}$ (1936) também descreve a muda embriônica de $R$. prolixus e, citando Galliard, afirma que a muda embriônica é uma verdadeira muda, pois a cutícula possui espinhos e cada apêndice é envolvido separadamente.

O estudo das exúvias de $T$. circummaculata e $T$. rubrovaria feitas por microscopia eletrônica de varredura confirmam as referências anteriores, pois nelas podem ser percebidas as antenas, o labro, três pares de pernas em formação e espinhos (Figuras 2d, 3f).

\section{AGRADECIMENTOS}

À Fundação Nacional de Saúde, seccional do Rio Grande do Sul, pelo apoio na coleta de campo e pela infra-estrutura oferecida.

Tabela 2 - Comparações estatísticas referentes às mensurações de largura e de comprimento de ovos e cascas de ovos de T. circummaculata e T. rubrovaria.

\begin{tabular}{|c|c|c|c|c|}
\hline \multirow[t]{2}{*}{ Comparações } & \multicolumn{2}{|c|}{ Comprimento } & \multicolumn{2}{|c|}{ Largura } \\
\hline & "t" observado & Resultado & "t" observado & Resultado \\
\hline $\begin{array}{l}\text { O vos de T. circummaculata e T. rubrovaria } \\
\text { Cascas de ovos de T. circummaculata x T. rubrovaria } \\
\text { O vos x Cascas de ovos de T. circummaculata } \\
\text { O vos x Cascas de ovos de T. rubrovaria }\end{array}$ & $\begin{array}{r}16,77 \\
18,40 \\
5,09 \\
4,20\end{array}$ & $\begin{array}{l}\text { Significante } \\
\text { Significante } \\
\text { Significante } \\
\text { Significante }\end{array}$ & $\begin{array}{r}27,39 \\
17,13 \\
4,74 \\
1,44\end{array}$ & $\begin{array}{l}\text { Significante } \\
\text { Significante } \\
\text { Significante } \\
\text { Não significante }\end{array}$ \\
\hline
\end{tabular}

Nota: Valor crítico da estatística "t" para todas as comparações: 1,99 (78 graus de liberdade). 


\section{REFERÊNCIAS}

1. Abalos JW, Wigodzinsky P. Las Triatominae argentinas (Reduviidae, Hemiptera). Tucumán: U niversidade Nacional de Tucumán/Instituto de Medicina Regional; 1951. (Publicación, 601; Monografia, 2).

2. Ayres M, Ayres Júnior M. A plicações estatísticas em BASIC. São Paulo: McGraw-Hill; 1987.

3. Barata JMS. Aspectos morfológicos de ovos de Triatominae. II. Características macroscópicas e exocoriais de dez espécies do gênero Rhodnius Stal, 1859 (Hemiptera-Reduviidae). Rev Saúde Pública 1981;15:490-542.

4. Carcavallo RU, Martinez A. Biologia, ecologia y distribucion geografica de los triatominos americanos. Chagas 1985;1(N esp):149-208.

5. Carcavallo RU, Canale DM, Martinez A. Habitats de triatominos argentinos y zonas ecologicas donde prevalecen. Chagas 1988;5:8-17.

6. Franca Rodrígues M, Lindner $C$, Salvattela R, Fernández RL, M oreira LP. Importancia médica y ecologia de los triatomíneos de la República O riental del U ruguay. Res J Cienc $N$ at 1980;1:35-6.

7. Galliard H. Recherches sur les reduvidés hématophages Rhodnius et Triatoma. VIII. Le dévelopment de I'oeuf des triatomes a température constante. Ann Parasitol H um Comp 1936;14:97-112.

8. Gonçalves TCM , Jurberg J, Costa JM, Souza W. Estudo morfológico comparativo de ovos e ninfas de Triatoma maculata (Erichson, 1848) e Triatoma pseudomaculata Corrêa e Espínola, 1964 (Hemiptera, Reduviidae, Triatominae). Mem Inst O swaldo Cruz 1985;80:263-76.

9. Haridass ET. U Itraestructure of the eggs of Reduviidae: III. Eggs of Triatominae and Echtrichodiinae (InsectaHeteroptera). Proc Indian Acad Sci (Anim Sci) 1986;95:447-56.
10. Jurberg J, Gonçalves TCM, Costa JM, Souza W. Contribuição ao estudo morfológico de ovos e ninfas de Triatoma brasiliensis N eiva, 1911 (Hemiptera, Reduviidae, Triatominae). Mem Inst O swaldo Cruz 1986;81:111-20.

11. Lent $\mathrm{H}$, Wygodzinsky P. Revision of the Triatominae (Hemiptera, Reduviidae), and significance as vectores of Chagas' disease. Bull Am Mus N at Hist 1970;163:313-5

12. Mellanby $\mathrm{H}$. The later embryology of Rhodnius prolixus. Q J Microsc Sci 1936;79:1-41.

13. Perlowagora-Szumlewicz A, Muller CA, Moreira CJC. Studies in search of a suitable experimental insect model for xenodianosis of hosts with Chagas'disease. 3. On the interaction of vector species and parasite strain in the reaction of bugs to infection by Trypanosoma cruzi. Rev Saúde Pública 1988;22:390-400.

14. Rosa JA, Barata JMS, Barelli N, Santos JLF, Belda N eto FM. Sexual distinctions between $5^{\text {th }}$ instar nymphs of six species of Triatominae (Hemiptera, Reduviidae). Mem Inst O swaldo Cruz 1992;87:257-64.

15. Rosa JA. Contribuição ao estudo morfológico de ovos e ninfas de $1^{\circ} \mathrm{O} e$ de $5^{\circ}$ estádios de Triatoma circummaculata (Stal, 1859) e de Triatoma rubrovaria (Blanchard, 1843) (Hemiptera, Roduviidae). [Tese]. São Paulo: Instituto de Ciências Biomédicas da Universidade de São Paulo; 1995.

16. Silva IG. Influência da temperatura na biologia de Triatomíneos. I - Triatoma rubrovaria (Blanchard, 1843) (Hemiptera, Reduviidae). Rev Goiana Med 1985;31:1-37.

17. Williams CB, Buxton BA. IV. On the biology of Sphodromantis guttata (M antidae). Trans R Entomol Soc Lond 1916;86:86-104. 